\title{
Multi-Stage 20-m Shuttle Run Fitness Test, Maximal Oxygen Uptake and Velocity at Maximal Oxygen Uptake
}

\author{
by \\ Giorgos P. Paradisis ${ }^{1}$, Elias Zacharogiannis ${ }^{1}$, Dafni Mandila ${ }^{1}$, Athanasia Smirtiotou${ }^{1}$, \\ Polyxeni Argeitaki ${ }^{1}$, Carlton B Cooke ${ }^{2}$
}

The multi-stage 20-m shuttle run fitness test (20mMSFT) is a popular field test which is widely used to measure aerobic fitness by predicting maximum oxygen uptake $\left(V_{2} m a x\right)$ and performance. However, the velocity at which $\mathrm{VO}_{2}$ max occurs (vVO2max) is a better indicator of performance than $\mathrm{VO}_{2}$ max, and can be used to explain interindividual differences in performance that $\mathrm{VO}_{2}$ max cannot. It has been reported as a better predictor for running performance and it can be used to monitor athletes' training for predicting optimal training intensity. This study investigated the validity and suitability of predicting $\mathrm{VO}_{2}$ max and $v V \mathrm{O}_{2}$ max of adult subjects on the basis of the performance of the 20mMST. Forty eight (25 male and 23 female) physical education students performed, in random order, a laboratory based continuous horizontal treadmill test to determine $V_{2} \mathrm{max}_{\text {, }} \mathrm{vV \textrm {O } _ { 2 } m a x}$ and a 20mMST, with an interval of 3 days between each test. The results revealed significant correlations between the number of shuttles in the $20 m M S F T$ and directly determined $V_{2} \max (r=0.87, p<0.05)$ and $v \mathrm{VO}_{2} \max (r=0.93, p<0.05)$. The equation for prediction of $V O_{2}$ max was $y=0.0276 x+27.504$, whereas for $v V O_{2}$ max it was $y=0.0937 x+6.890$. It can be concluded that the 20mMSFT can accurately predict $V_{2}$ max and vVO max and this field test can provide useful information regarding aerobic fitness of adults. The predicted $v V O_{2} m a x$ can be used in monitoring athletes, especially in determining optimal training intensity.

Key words: aerobic capacity, prediction, testing.

\section{Introduction}

Maximal oxygen uptake $\left(\mathrm{VO}_{2} \max \right)$ has been characterized as an important indicator for success in distance running performance as well as for health (Astrand and Saltin, 1967; Hamlin et al., 2012; Noakes et al., 1990). However, the velocity at which $\mathrm{VO}_{2} \max$ occurs $\left(\mathrm{vVO}_{2} \max \right)$ is a better indicator of performance than $\mathrm{VO}_{2} \max$, as it effectively combines both $\mathrm{VO}_{2} \max$ and running economy in one term (di Prampero et al., 1986) and can also be used to explain inter-individual differences in performance that $\mathrm{VO}_{2}$ max or running economy alone cannot (Billat and Koralsztein, 1996;
Billat et al., 2000; Jones and Carter, 2000). Compared to $\mathrm{VO}_{2} \max , \mathrm{vVO}_{2} \max$ is a better predictor for middle and long-distance running performance (Noakes et al., 1990) and it has been reported that it should be used to monitor athletes' training and to determine optimal training intensity for distance runners (Billat and Koralsztein, 1996; Billat et al., 2000; Laursen and Jenkins, 2002; Smith et al., 1999). Additionally, it seems that $\mathrm{vVO}_{2} \max$ is the minimum speed required to elicit athlete's $\mathrm{VO}_{2}$ max, and it is necessary to train at this speed in order to improve $\mathrm{VO}_{2}$ max and thus aerobic capacity (Billat,

\footnotetext{
1 - Department of Athletics, Faculty of Physical Education \& Sports Science, National E Kapodistrian University of Athe ns, Greece.

2 - Carnegie Faculty of Sport and Education, Leeds Beckett University, UK.
} 
2001; Billat and Koralsztein, 1996; Billat et al., 2000; Hill and Rowell, 1997; Jones and Carter, 2000). Maximizing $\quad \mathrm{VVO}_{2} \max$ through training will increase the running speeds which correspond to a given percentage of $\mathrm{VO}_{2} \max$ and will improve performance since athletes tend to use similar percentages of $\mathrm{VO}_{2}$ max for a given exercise duration (Jones and Carter, 2000). Thus, $\mathrm{vVO}_{2} \max$ can be very useful for the determination of the intensity of training programs. $\mathrm{vVO}_{2} \mathrm{max}$ is directly measured within a laboratory based test during which running speed increases gradually until the athlete reaches $\mathrm{VO}_{2} \max$ (Billat and Koralsztein, 1996). The direct measurements of $\mathrm{VO}_{2}$ max and $\mathrm{vVO}_{2} \mathrm{max}$ in a laboratory are time consuming (only one subject at a time) and require relatively expensive equipment, trained personnel and may not be appropriate for some applications. Consequently, there is a demand for quick, inexpensive and valid field tests that can provide a reasonable estimate of $\mathrm{VO}_{2}$ max. The multi-stage 20$\mathrm{m}$ shuttle run fitness test (20mMSFT) was developed by Léger and Lambert (1982) and it has been used widely since its introduction. The 20mMSFT is a popular field test which is used among athletes of all levels, from children to elite athletes. The 20mMSFT has been used widely for distance runners, soccer players, basketball players, squash, karate and even ice-hockey players in order to measure their aerobic fitness and to predict performance (Paliczka et al., 1987; Ramsbottom et al., 1988; Koklu et al., 2011; Koropanovski et al., 2011; Aslan, 2013). Several studies demonstrated that the assessment of aerobic fitness with the 20mMSFT on a regular basis is beneficial for evaluating the effectiveness of training programs (Castagna et al., 2006; Castagna et al., 2006; Impellizzeri et al., 2005), monitoring soccer players (Castagna et al., 2006), investigating seasonal variations in physiological variables of soccer players (Caldwell and Peters, 2009), basketball players (Ostojic et al., 2006) and ice-hockey players (Geithner et al., 2006). Many studies have reported high correlations $(0.90-0.93)$ between performance in the 20mMSFT and $\mathrm{VO}_{2}$ max (Léger and Lambert, 1982; Paliczka et al., 1987; Ramsbottom et al., 1988; Sproule et al., 1993; Wilkinson et al., 1999). However, as the only outcome of the $20 \mathrm{mMSFT}$ is predicted $\mathrm{VO}_{2} \max$, there is no information regarding $\mathrm{vVO}_{2}$ max, and consequently no detail on an optimal training intensity for improving performance (Billat and Koralsztein, 1996). The aim of this study was to investigate the validity and suitability of predicting both $\mathrm{VO}_{2}$ max and the $\mathrm{vVO}_{2} \mathrm{max}$ of adult subjects taking into account performance of the 20mMSFT. As the correlations between $\mathrm{VO}_{2}$ max and $\mathrm{vVO}_{2}$ max (Billat and Koralsztein, 1996) and between the 20mMSFT and $\mathrm{VO}_{2}$ max have been reported as greater than $\mathrm{r}=0.9$, it was hypothesized that the correlation between $\mathrm{vVO}_{2} \mathrm{max}$ and the 20mMSFT should also be of similar magnitude, which should facilitate the product of a predictive equation.

\section{Material and Methods}

Forty eight (25 male and 23 female) PE college students (age $=21.20 \pm 1.91$ years; body mass $=66.07 \pm 11.22 \mathrm{~kg}$; body height $=1.72 \pm 0.10 \mathrm{~m}$ and $\%$ of body fat $=17.40 \pm 5.32 \%$ ) performed, in random order, a laboratory based continuous horizontal treadmill test to determine $\mathrm{VO}_{2}$ max and a 20mMST, with an interval of 3 days between each test. Testing was performed at the same hour of day $\pm 2 \mathrm{~h}$, with subjects instructed to consume a light meal at least 4 hours before testing and to avoid intense exercise in the preceding 24 hours.

Each subject's percent body fat was estimated for descriptive purposes using a Harpenden skin-fold caliper (model 68875, UK). Skinfold sites included bicep, tricep, subscupular and suprailiac (Durnin and Womersley, 1974). Prior to participation, subjects received information regarding the design of the study and then gave written informed consent. Prior to the commencement of the research, all procedures involved in this investigation were reviewed and approved by the Athens University's Research Ethics Committee.

On their initial visit to the laboratory, all subjects were familiarized with the procedures for the treadmill test (Run race 1200, Technogym, Cesena Italy) and for the 20mMSFT. Two days later, participants completed an incremental test to volitional exhaustion with a starting velocity (set during familiarization) of $7-12 \mathrm{~km} \cdot \mathrm{h}^{-1}$ and $1 \%$ slope, for the determination of $\mathrm{VO}_{2}$ max or $\mathrm{VO}_{2}$ peak (dependent on meeting criteria for $\mathrm{VO}_{2}$ max or not) and $\mathrm{vVO}_{2}$ max or $\mathrm{vVO}_{2}$ peak in an air-conditioned laboratory with the temperature set at $19-21^{\circ} \mathrm{C}$. Treadmill speed was calibrated previously with a subject running at different speeds while the time it takes for the completion for 30 treadmill 
revolutions was recorded on a stop watch. Following a 5-min warm-up, the velocity was increased by $1 \mathrm{~km} \cdot \mathrm{h}^{-1}$ every $3 \mathrm{~min}$ from their individual starting velocity until volitional exhaustion. Gas collection was made during the last $60 \mathrm{~s}$ period of each $3 \mathrm{~min}$ stage in order to allow the subject to attain steady state $\mathrm{VO}_{2}$ (Lafontaine et al., 1981). $\mathrm{VO}_{2}$ was measured by the open circuit Douglas Bag method. The subject breathed through a low resistance 2-way Hans-Rudolph 2700 B valve (Shawnee, USA). The expired gases passed through a $90 \mathrm{~cm}$ length of $340 \mathrm{~mm}$ diameter flexible tubing in to 200-liter capacity Douglas Bags. The concentration of $\mathrm{CO}_{2}$ and $\mathrm{O}_{2}$ in the expired air were measured by the GIR 250 Hitech combined Oxygen and Carbon Dioxide Analyzer (Luton, England). The gas analyzers were calibrated against standardized gases $\left(15.35 \% \mathrm{O}_{2}, 5.08 \% \mathrm{CO}_{2}\right.$ and $100 \% \mathrm{~N}_{2}$ ). Expired volume was measured by means of a dry gas meter (Harvard) previously calibrated with a 3-liter syringe. Barometric pressure and gas temperature were recorded and respiratory gas exchange data for each work load (i.e. $\mathrm{VO}_{2}, \mathrm{VCO}_{2}$ VE and RER) were determined on a locally developed computer program based on the computations described by McArdle et al. (2010), when VEatps, $\mathrm{FECO}_{2}$ and $\mathrm{FEO}_{2}$ are known. The highest $\mathrm{VO}_{2}$ value obtained during an incremental exercise test was recorded as the subject's $\mathrm{VO}_{2}$ peak which also elicited a heart rate within $\pm 10 \mathrm{bpm}$ of age predicted HRmax and a Respiratory Exchange Ratio (RER) greater than 1.05.

The lowest running speed that elicits a $\mathrm{VO}_{2}$ equivalent to $\mathrm{VO}_{2}$ peak during the treadmill test was defined as $\mathrm{vVO}_{2}$ peak (Billat and Koralsztein, 1996). If the final exercise work load was not completed for $120 \mathrm{~s}$ but $\mathrm{VO}_{2}$ was increased then $\mathrm{vVO}_{2}$ peak was determined from the following equation (Kuipers et al., 1985):

\section{$\mathrm{vVO}_{2}$ peak = last work load completed in $120 \mathrm{~s}+$ [(time of the uncompleted work load $\left./ \mathbf{1 2 0})^{*} 1\right]$}

Fingertip blood samples were taken within 5 minutes of the completion of the test for the determination of lactate levels. To avoid sweat contamination the first drop of blood was wiped off, and only the second was used for analysis.
The concentration of lactate was measured enzymatically (Dr Lange, Cuvette Test LKM 140, Hamburg, Germany) using a miniphotometer LP 20 Plus (Dr Lange, Hamburg, Germany). Blood was taken using $10 \mu \mathrm{l}$ end-to-end capillaries and placed in a reagent solution hemolyzing the blood. Lactate was processed in a reaction producing quinonimin in proportion to the amount of lactate in the sample, and the concentration of quinonimin was read off in an LP 20 Plus apparatus at $540 \mathrm{~nm}(576 \mathrm{THz})$ after a 3 min reaction time.

The heart rate (HR) was recorded every $5 \mathrm{~s}$ throughout the exercise tests using short-range telemetry (Polar S 710, Polar, Helsinki, Finland).

The 20mMSFT (Handbook, 1983) was administered in a sports hall (temperature $19-21^{\circ}$ C). It involved running between two lines set $20 \mathrm{~m}$ apart at a pace dictated by a recording emitting tones at appropriate intervals. Velocity was 8.5 $\mathrm{km} \cdot \mathrm{h}^{-1}$ for the first minute, which increased by 0.5 $\mathrm{km} \cdot \mathrm{h}^{-1}$ every minute thereafter. The test score achieved by the subject was the number of $20 \mathrm{~m}$ shuttles completed before the subject either withdrew voluntarily from the test, or failed to be within $3 \mathrm{~m}$ of the end lines on two consecutive tones. Heart rates were continuously recorded throughout the test (Polar heart rate monitor S 710), whereas blood lactate was collected $5 \mathrm{~min}$ after the test. Scores from the treadmill test and the 20mMSFT were compared using a paired $t$ test. Data were assessed for normality (Kolomonov and Smirnoff) and the relationships between variables for the two tests were calculated using the Pearson's product moment correlation coefficient. Significance was set at $\mathrm{p}<0.05$.

\section{Results}

The analysis of the data revealed statistically significant correlations between the number of shuttles in the 20mMSFT and treadmill $\mathrm{VO}_{2} \max (\mathrm{r}=$ $0.87, \mathrm{p}<0.05$; Figure 1$)$ as well as $\mathrm{vVO}_{2} \max (\mathrm{r}=0.93$, $\mathrm{p}<0.05$; Figure 2). Additionally, there were no significant differences between the measured and predicted values of $\mathrm{VO}_{2} \max (49.98 \pm 8.33$ and 49.97 $\left.\pm 7.17 \mathrm{ml} \cdot \mathrm{kg}^{-1} \cdot \mathrm{min}^{-1}\right), \mathrm{vVO}_{2} \max (14.52 \pm 2.65$ and $\left.14.51 \pm 2.43 \mathrm{~km} \cdot \mathrm{h}^{-1}\right), \mathrm{HRVO}_{2} \max (194.1 \pm 10.03$ and $195.2 \pm 7.50$ beats $\left.\cdot \mathrm{min}^{-1}\right)$ and LacuteVO $2 \max (12.05 \pm$ 1.96 and $\left.12.09 \pm 1.90 \mathrm{mmol} \cdot \mathrm{L}^{-1}\right)$. The equation for prediction of $\mathrm{VO}_{2} \max$ was $\mathrm{y}=0.0276 \mathrm{x}+27.504$, whereas for the $\mathrm{vVO}_{2}$ max it was $\mathrm{y}=0.0937 \mathrm{x}+6.890$. 


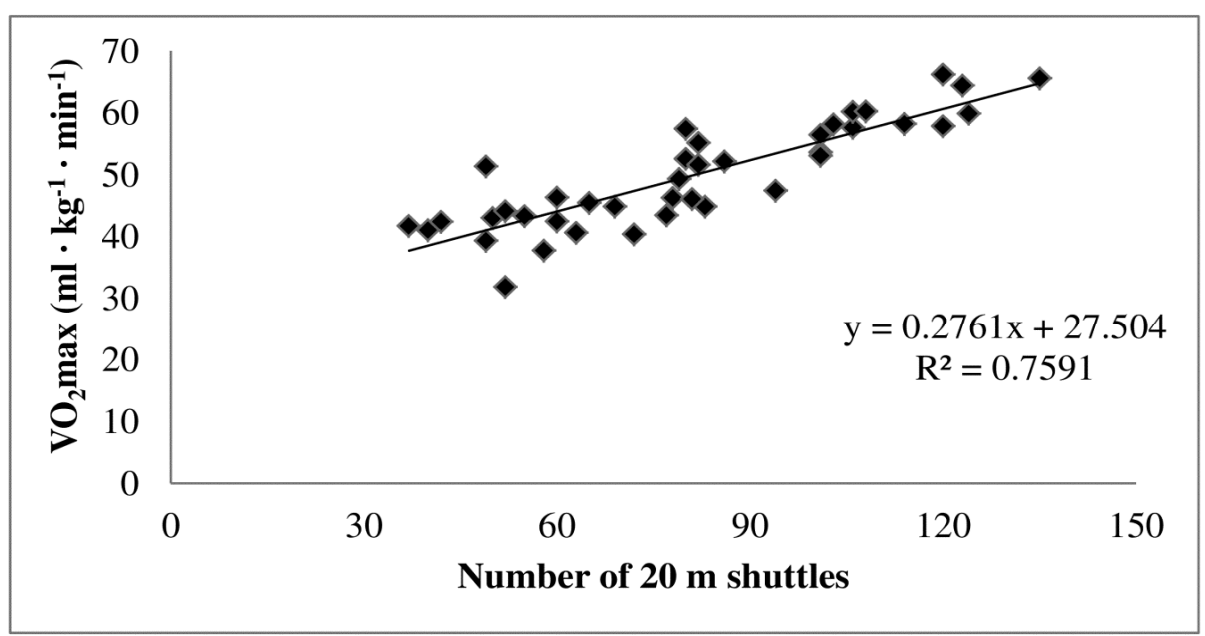

Figure 1

The relationship between the number of multi-stage 20-m shuttle run fitness test and direct measurement of maximal oxygen uptake $(n=48)$.

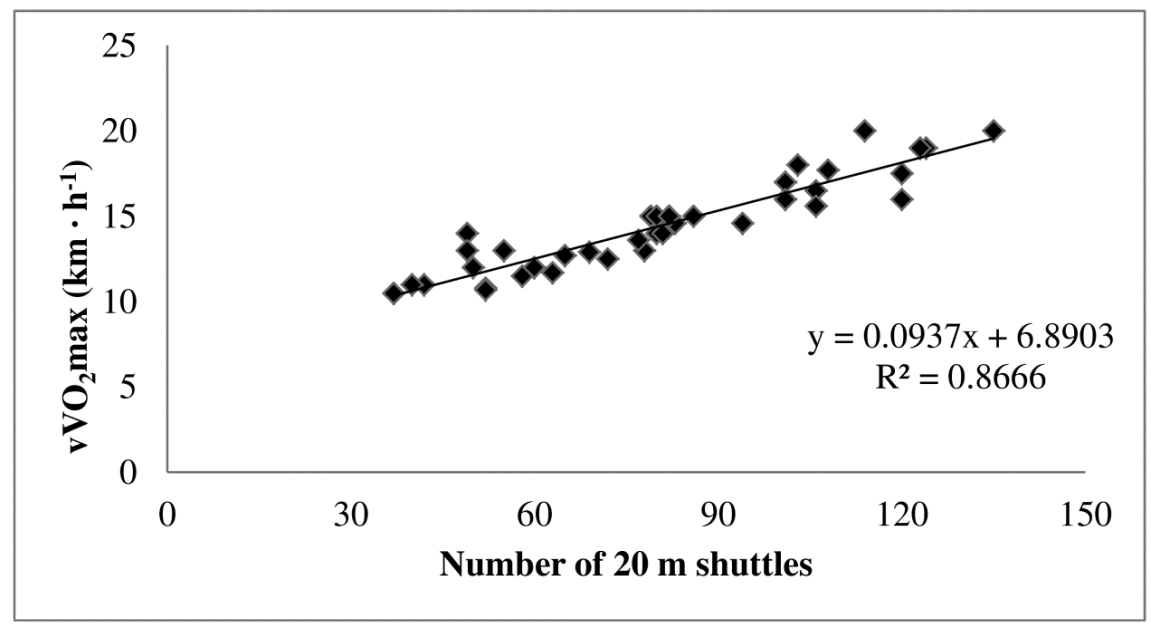

Figure 2

The relationship between the number of multi-stage 20-m shuttle run fitness test and direct measurement of the velocity at maximal oxygen uptake $(n=48)$ 


\section{Discussion}

The purpose of the present study was to investigate the validity and suitability of predicting $\mathrm{VO}_{2}$ max and $\mathrm{vVO}_{2}$ max of adult subjects on the basis of performance of the 20mMSFT. The results indicated a high correlation coefficient between shuttles in the 20mMSFT and $\mathrm{VO}_{2}$ max as well as $\mathrm{vVO}_{2}$ max. Ideally this type of research should include a two sub-samples design, where one subsample is used to obtain the estimation equations and the other sub-sample is used to validate the equations (Morais et al., 2011). However, it can be argued that the present study design is appropriate, as several publications have adopted the same research design (Sproule et al., 1993; Ramsbottom et al., 1988; Paliczka et al., 1987; Stickland et al., 2003; Léger et al., 1988).

The regression model proposed in the present study for the prediction of $\mathrm{VVO}_{2} \mathrm{max}$ is appropriately accurate as indicated the $\mathrm{R}^{2}$, the adjusted $R^{2}$ and the standard error of estimation $\left(R^{2}\right.$ $=0.867, \mathrm{R}^{2}$ adj $=0.863$ and $\sigma_{\text {est }}=0.980$ ), with only $13 \%$ of the variation not explained by this prediction model. While $13 \%$ is quite small, some caution should be exercised when this prediction model is used. Similarly, the model proposed in the present study for the prediction of $\mathrm{VO}_{2} \max$ is also appropriately accurate $\left(\mathrm{R}^{2}=0.759, \mathrm{R}^{2}\right.$ adj $=0.753$ and $\left.\sigma_{\text {est }}=4.142\right)$, even though it is not as strong as the model for $\mathrm{vVO}_{2}$ max prediction. Again, this prediction model should be used with caution as $24 \%$ of the variation is not explained. The unexplained $13 \%$ and $24 \%$ of variation could be due to factors such as the contribution of anaerobic power and capacity, and/or by some technical error and biological variability.

The correlation between the 20mMSFT shuttles and $\mathrm{VO}_{2}$ max reported in the present study was similar to values from other studies for adults (Léger and Lambert, 1982; Paliczka et al., 1987; Ramsbottom et al., 1988; Sproule et al., 1993; Wilkinson et al., 1999) but was higher than those for children (Van Mechelen et al., 1986). Statistical analysis also indicated that there were no differences between the direct and indirect values of $\mathrm{VO}_{2}$ max. These results confirmed the use of the 20mMSFT as a valid predictor of $\mathrm{VO}_{2}$ max with reasonable accuracy (Paliczka et al., 1987). The results of the present study produced a higher correlation between shuttles on the 20mMSFT and $\mathrm{vVO}_{2}$ max compared to $\mathrm{VO}_{2} \max$ ( $\mathrm{r}=0.93$ and $\mathrm{r}=0.87$, respectively). Additionally, statistical analysis indicated that there were no differences between the direct and indirect values of $\mathrm{vVO}_{2}$ max. As this is the first study correlating shuttles in the 20mMSFT and $\mathrm{vVO}_{2 m a x}$, there are no comparable data available in the literature. However, Paliczka et al. (1987) correlated the 20mMSFT and performance in a $10 \mathrm{~km}$ run and reported a similar correlation $(\mathrm{r}=0.93)$.

The prediction of $\mathrm{vVO}_{2} \max$ is very useful, especially when it occurs simultaneously for a group of participants through a simple and inexpensive test such as the 20MSFT. Several studies have demonstrated that for well-trained athletes $\mathrm{VVO}_{2} \mathrm{max}$ is the lowest exercise intensity necessary to reach their $\mathrm{VO}_{2}$ max during a workout, improving their $\mathrm{VO}_{2} \mathrm{max}$ and therefore, aerobic capacity (Billat, 2001; Billat and Koralsztein, 1996; Billat et al., 2000; Hill and Rowell, 1997; Jones and Carter, 2000; Bragada et al., 2010). Moreover, interval training at high intensities, such as $\mathrm{vVO}_{2}$ max, allows athletes to maintain $\mathrm{VO}_{2}$ max for a prolonged period of time and leads to a relatively greater improvement in $\mathrm{VO}_{2}$ max (Demarie et al., 2000). Furthermore, maximizing $\mathrm{vVO}_{2}$ max through training increases the running speed which corresponds to a given percentage of $\mathrm{VO}_{2}$ max (Jones and Carter, 2000). This is very important for improving performance since athletes tend to use similar percentages of $\mathrm{VO}_{2}$ max for a given exercise duration (Jones and Carter, 2000).

The prediction models for the evaluation of $\mathrm{vVO}_{2}$ max and $\mathrm{VO}_{2} \max$, aerobic fitness and performance using the 20mMSFT can be used as appropriately accurate tools when direct measurements in a laboratory are not possible due to time and cost restrictions. The findings of the present study provide a relatively quick, inexpensive and valid field test that can provide a reasonable estimate of $\mathrm{vVO}_{2} \max$, which can be utilized in many different situations. However, some limitations, including the characteristics of the participants, have to be emphasized. The prediction models were derived from testing PE college students with relatively good aerobic fitness. Employment of these models with a different age group (youth or mature athletes), of different capabilities (sedentary, highly trained or world class athletes) and specific sports (e.g. soccer, track $\&$ field) has not been tested and the predictive accuracy for these specific situations remains unknown. 
It can be concluded that the $20 \mathrm{~m}$ multistage shuttle run test can accurately predict $\mathrm{VO}_{2}$ max and $\mathrm{vVO}_{2}$ max corresponding to maximal oxygen uptake and this field test can provide useful information regarding the aerobic fitness of adults. Additionally, the predicted $\mathrm{vVO}_{2}$ max from this test could be used to monitor athletes and determine optimal training intensity in order to improve $\mathrm{VO}_{2}$ max and thus, aerobic capacity.

\section{References}

Aslan A. Cardiovascular Responses, Perceived Exertion and Technical Actions During Small-Sided Recreational Soccer: Effects of Pitch Size and Number of Players. J Hum Kinet, 2013; 38: 95-105

Astrand PO, Saltin B. Maximum oxygen uptake in athletes. J Appl Physiol, 1967; 23: 353-358

Billat LV. Interval training for performance: a scientific and empirical practice. Sports Med, 2001; 31(1): 13-31

Billat LV, Koralsztein JP. Significance of the velocity at $\mathrm{VO}_{2} \max$ and time to exhaustion at this velocity. Sports Med, 1996; 22(2): 90-108

Billat VL, Slawinski J, Bocquet V, Demarle A, Lafitte L, Chassaing P, Koralsztein JP. Intermittent runs at the velocity associated with maximal oxygen uptake enables subjects to remain at maximal oxygen uptake for a longer time than intense but submaximal runs. Eur J Appl Physiol, 2000; 81(3): 188-196

Bragada JA, Santos P, Maia JA, Colaco P, Lopes VP, Barbosa TM. Longitudinal study in 3000m male runners: relationship between performance and physiological parameters. J Sport Sci Med, 2010; 9: 439-444

Caldwell BP, Peters DM. Seasonal variation in physiological fitness of a semiprofessional soccer team. $J$ Strength Cond Res, 2009; 23(5): 1370-1377

Castagna C, Impellizzeri FM, Belardinelli R, Abt G, Coutts A, Chamari K, D'Ottavio S. Cardiorespiratory responses to Yo-yo Intermittent Endurance Test in nonelite youth soccer players. J Strength Cond Res, 2006; 20(2): 326-330

Castagna C, Impellizzeri FM, Chamari K, Carlomagno D, Rampinini E. Aerobic Fitness and Yo-Yo Continuous and Intermittent Tests Performances in Soccer Players: Acorrelation Study. J Strength Cond Res, 2006; 20(2): 320-325

Demarie S, Koralsztein J, Billat V. Time limit and time at VO2max'during a continuous and an intermittent run. J Sport Med Phys Fit, 2000; 40(2): 96-102

di Prampero PE, Atchou G, Bruckner JC, Moia C. The energetics of endurance running. Eur J Appl Physiol, 1986; 55(3): 259-266

Durnin J, Womersley J. Body fat assessed from total body density and its estimation from skinfold thickness: measurements on 481 men and women aged from 16 to 72 years. Br J Nutr, 1974; 32(1): 77-97

Geithner CA, Lee AM, Bracko MR. Physical and performance differences among forwards, defensemen, and goalies in elite women's ice hockey. J Strength Cond Res, 2006; 20(3): 500-505

Hamlin MJ, Draper N, Blackwell G, Shearman JP, Kimber NE. Determination of Maximal Oxygen Uptake Using the Bruce or a Novel Athlete-Led Protocol in a Mixed Population. J Hum Kinet, 2012; 31(1): 97104

Handbook EP. Testing Physical Fitness: London HMSO; 1983

Hill DW, Rowell A. Responses to exercise at the velocity associated with VOzmax. Med Sci Sports Exer, 1997; 29(1): 113-116

Impellizzeri FM, Rampinini E, Marcora SM. Physiological assessment of aerobic training in soccer. J Sports Sci, 2005; 23(6): 583-592

Jones AM, Carter H. The effect of endurance training on parameters of aerobic fitness. Sports Med, 2000; 29(6): 373-386

Köklü Y, Alemdaroğlu U, Koçak F, Erol A, Fındıkoğlu G. Comparison of chosen physical fitness characteristics of Turkish professional basketball players by division and playing position. J Hum Kinet, 2011; 30(1): 99-106

Koropanovski N, Berjan B, Bozic P, Pazin N, Sanader A, Jovanovic S, Jaric S. Anthropometric and physical performance profiles of elite karate kumite and kata competitors. J Hum Kinet, 2011; 30(1): 107-114

Kuipers H, Verstappen F, Keizer H, Geurten P, Van Kranenburg G. Variability of aerobic performance in the 
laboratory and its physiologic correlates. Int J Sports Med, 1985; 6(04): 197-201

Lafontaine T, Londeree BR, Spath WK. The maximal steady state versus selected running events. Med Sci Sports Exerc, 1981; 13(3): 190-192

Laursen PB, Jenkins DG. The scientific basis for high-intensity interval training: optimising training programmes and maximising performance in highly trained endurance athletes. Sports Med, 2002; 32(1): 53-73

Léger LA, Lambert J. A maximal multistage 20-m shuttle run test to predict $\backslash$ dot $\mathrm{VO}_{2}$ max. Eur J Appl Physiol, 1982; 49(1): 1-12

McArdle WD, Katch F, Katch V. Exercise physiology: nutrition, energy, and human performance. London: Lippincott Williams \& Wilkins, 121-130; 2010

Morais JE, Costa MJ, Mejias EJ, Marinho DA, Silva AJ, Barbosa TM. Morphometric study for estimation and validation of trunk transverse surface area to assess human drag force on water. J Hum Kinetics, 2011; 28: 5-13

Noakes T, Myburgh K, Schall R. Peak treadmill running velocity during the $\mathrm{VO}_{2}$ max test predicts running performance. J Sports Sci, 1990; 8(1): 35-45

Ostojic SM, Mazic S, Dikic N. Profiling in basketball: physical and physiological characteristics of elite players. J Strength Cond Res, 2006; 20(4): 740-744

Paliczka V, Nichols A, Boreham C. A multi-stage shuttle run as a predictor of running performance and maximal oxygen uptake in adults. Brit J Sport Med, 1987; 21(4): 163-165

Ramsbottom R, Brewer J, Williams C. A progressive shuttle run test to estimate maximal oxygen uptake. $\mathrm{Br} J$ Sport Med, 1988; 22(4): 141-144

Smith TP, McNaughton LR, Marshall KJ. Effects of 4-wk training using Vmax/Tmax on $\mathrm{VO}_{2}$ max and performance in athletes. Med Sci Sports Exer, 1999; 31(6): 892-896

Sproule J, Kunalan C, McNeill M, Wright H. Validity of 20-MST for predicting $\mathrm{VO}_{2}$ max of adult Singaporean athletes. Br J Sport Med, 1993; 27(3): 202-204

Van Mechelen W, Hlobil H, Kemper H. Validation of two running tests as estimates of maximal aerobic power in children. Eur J Appl Physiol, 1986; 55(5): 503-506

Wilkinson DM, Fallowfield JL, Myers SD. A modified incremental shuttle run test for the determination of peak shuttle running speed and the prediction of maximal oxygen uptake. J Sports Sci, 1999; 17(5): 413419

\section{Corresponding author:}

\section{Giorgos Paradisis}

Department of Athletics, Faculty of Physical Education \& Sports Science, National \& Kapodistrian University of Athens, Greece

Ethnikis Antistasis 41, Dafni, Athens, 172 37, Greece

Phone: +30 2107276102

E-mail: gparadi@phed.uoa.gr

(c) Editorial Committee of Journal of Human Kinetics 